\title{
REVISIONES
}

\section{Motricidad Humana y gestión municipal}

\author{
Human Kinetics and Municipal Managment \\ Motricidade Humana e gestão municipal
}

\author{
José Pazos-Couto, ${ }^{\text {a Eugenia Trigo }}{ }^{b}$
}

aUniversidad de Vigo, Facultade de Ciencias da Educación e do Deporte de Pontevedra, Campus a Xunq, España. Telf.: 34 627589680. Correo electrónico: Correo electrónico: chema3@uvigo.es bInstituto internacional del Saber-Colombia. Correo electrónico: etrigoa@gmail.com

\section{RESUMEN}

El presente trabajo muestra una visión de cómo afrontar desde la Motricidad el campo de la gestión deportiva en el ámbito de la administración local, destacando diversas cuestiones como su conceptualización, el deporte, la dimensión pedagógica de la Motricidad Humana, la gestión y el perfil profesional. Acabamos mostrando una visión actualizada de la Motricidad y sus perspectivas.

Palabras clave: políticas públicas, motricidad, gestión, administración local.

\section{ABSTRACT}

The present article shows how to deal with the field of sports management from the point of view of human movement in local administration; emphasizing various questions such as its conceptualization, sport, the educational dimension of human movement, management, and the professional profile. The article ends with an updated view of human movement and its perspectives.

Key words: public policies, human movement, management, local administration.

\section{RESUMO}

Apresenta-se uma visão de como enfrentar, a partir da Motricidade, o campo da gestão esportiva no âmbito da administração local, destacando-se diversas questões como sua conceituação, o esporte, a dimensão pedagógica da Motricidade Humana, a gestão, o perfil profissional e, conclui-se, apresentando, um estudo atualizado da Motricidade e suas perspectivas.

Palavras chave: políticas públicas, motricidade, gestão, administração local. 


\section{MOTRICIDAD HUMANA Y CIENCIA DE LA MOTRICIDAD HUMANA}

La Motricidad puede responder a dos acepciones: una a la capacidad que tenemos las personas de manifestarnos en el mundo de forma intencional y consciente, buscando trascender, superarnos, con sentido lúdico y desarrollo personal; es nuestra manifestación de lo que somos en el mundo. La otra hace referencia a la ciencia que estudia esa capacidad, que no es otra que la Ciencia de la Motricidad Humana (CMH) que describe y explica Manuel Sérgio; un concepto de ciencia que estudia al ser humano en esa búsqueda de la trascendencia a través de su corporeidad.

Para definir Motricidad, no podemos referirnos únicamente a un concepto, ya que también implica un posicionamiento personal y profesional, tanto en lo referente a la forma de entender al ser humano como en la forma de afrontar el trabajo con las personas. Además, el simple hecho de entender la Motricidad como un concepto amplio dentro de un paradigma emergente implica la necesidad de plantear nuevos debates, nuevos conceptos y nuevas formas de afrontar la praxis.

De esta forma, llegamos al paradigma emergente de la $\mathrm{CMH}$ que describe Manuel Sérgio desde un estudio de la Creatividad Motriz, en el que ya hablábamos de otra forma de entender la Educación Física y el Deporte, sin renegar de ellos en ningún momento, pero sí matizando nuestra forma de posicionarnos ante sus diferentes manifestaciones (deporte, gestión, salud, docencia, juego, circo, etc.).

Asumimos desde el principio que un posicionamiento epistemológico que tome en consideración la Motricidad implica que ésta se haya comprometido con el sentido de la vida, no sólo con el pensamiento sobre ésta, sino con ella como proceso y producto de la acción intencional. Entendemos que cuando la Ciencia de la Motricidad Humana surge, desaparece naturalmente la idea clásica y tradicional de Educación Física (Feitosa, Kolyniak, y Rath, 2006), tratando de huir en todo momento de la concepción dualista del ser humano, aunque sin dejar de lado ninguna de las manifestaciones de la Motricidad.

Comprendemos a su vez que la Motricidad Humana, "es un campo de conocimiento en cuyo interior (metafóricamente) hay unos constitutivos que están en una tensión permanente, y que constantemente tienen que revalidar su posición (su capital dentro del campo); dado que los campos se mantienen en constante redefinición" (Benjumea, 2010: 198).

Por ello, cuando hablamos de Motricidad nos referimos a la expresión total del ser humano en su existencia consciente, pues no es sólo en el juego, en el deporte o en las actividades de tiempo libre ni en las comúnmente conocidas como saludables, en las que ponemos de manifiesto nuestra Motricidad. En cada momento, en cada acción que realizamos de forma consciente e intencional, nos estamos haciendo presentes y con ello estamos manifestando nuestra corporeidad y Motricidad.

El tema de la "consciencia" es punto clave en la CMH. Basándonos en diversos investigadores de la Biología y Neurofenomenología actuales, muchos "mitos" se van destapando y, al mismo tiempo, se abre un campo nuevo a la investigación y a la praxis en la propia CMH. Estos son aspectos a tener en cuenta a la hora de "pensar" cualquier proyecto en relación a una comunidad de vida, como es un Ayuntamiento.

Este posicionamiento parte de la idea de que una epistemología de la Educación Física, o sea, la organización del conocimiento de la impropiamente denominada Educación Física, no se puede olvidar que ésta no es un área de físicos, sí de personas en el movimiento intencional de transcendencia (o superación) como lo entiende Sérgio (2008). 
La comprensión de un determinado objeto de estudio es fundamental para que diferentes investigadores puedan compartir dicha comprensión y trabajar de forma organizada para la construcción y difusión del conocimiento acerca de tal objeto. En el caso de la Motricidad, coincidiendo con Manuel Sérgio (1999), defendemos que ésta constituye:

Una energía... que es estatuto ontológico, vocación y provocación de apertura a la transcendencia.

El proceso adaptativo, a un medio ambiente variable, de un ser no especializado y, por eso, en el cual el ritmo evolutivo, incluyendo las estructuras del sistema nervioso central es lento, implicando la existencia de una demorada pubertad y de una familia estable y duradera.

El proceso evolutivo de un ser, como predisposición a la interioridad, a la práctica dialogal y a la cultura.

El proceso creativo de un ser en el que las prácticas lúdicas, agonísticas, simbólicas y productivas traducen la voluntad del hombre a realizarse como sujeto, o sea como autor responsable de sus actos (150)

Por ello ponemos énfasis en que "ser en el mundo" es vivir en movimiento intencional, siendo cuerpo consciente y comunicante. La existencia, a través de la Motricidad, es un continuo acto creativo; de un yo que implica hacer, saber, pensar, sentir, comunicar y querer (Kon-traste y Trigo, 1999). No hay ser humano sin la unidad de estos seis aspectos, pues no se puede hablar del ser humano si no se lo considera simultáneamente como un ser biológico, cultural, psicológico y social (Cyrulnik y Morin, 2005).

Corporeidad y Motricidad son dos conceptos que se complementan y necesitan, pues no puede existir uno sin el otro; en este sentido, hay abundantes autores que los consideran como conceptos equivalentes destacando el hecho de incluir este fenómeno en el contexto de las leyes más generales que tratan de explicar el universo como un todo (Brandão, 2001).

Somos cuerpo y es este cuerpo vivo el que manifiesta nuestra Motricidad, a través de la corporeidad, expresado en querer, sentir, pensar, hacer, compartir. Por tanto, el principal componente de la Motricidad, sin el cual ésta no existiría, es nuestra corporeidad, a la que atribuimos la condición de consciencia e intencionalidad.

Es en ella donde el cuerpo comunica las sensaciones y emociones, y es en ella donde vivimos nuestra realidad cotidiana. Como indica Merleau Ponty (1999), todo lo que sabe la ciencia o sabemos por nosotros mismos, se produce a través de nuestra experiencia y nuestras percepciones. Somos la fuente absoluta de nuestra existencia y quienes nos hacemos seres a nosotros mismos. Mediante la corporeidad somos nosotros en el mundo. Y las acciones, el deporte, las actividades lúdico recreativas, nuestro simple vivir consciente en ser, sentir, hacer, emocionar son manifestaciones más o menos visibles de nuestra Motricidad.

Es a partir de esta perspectiva que podemos poner en práctica las diferentes manifestaciones de la Motricidad y compartir nuestra Motricidad con la de los otros, o interaccionar con el medio en que vivimos: "en el momento en que damos un abrazo o resolvemos una ecuación, nuestra corporeidad actúa como unidad" (Bohórquez y Trigo, 2006: 12) y se manifiesta como un ser físico, emocional, mental, inconsciente, cultural, mágico e inmanente. Podemos afirmar que corporeidad y Motricidad se confunden por habitarse mutuamente. 


\section{MOTRICIDAD HUMANA, EDUCACIÓN FÍSICA Y DEPORTE}

La Motricidad nos sitúa ante una nueva concepción de las diversas manifestaciones del ser humano; y como tal supera el concepto actual de Educación Física. Además es un término multidimensional que entiende al ser humano como un ser complejo no divisible aunque, con diferentes dimensiones (psicológica, social, afectiva, motora) que ayudan a comprendernos y a manifestar nuestro ser.

Al respecto, coincidimos con Manuel Sérgio (2007: 11) cuando señala que "la Motricidad es el cuerpo en movimiento intencional, procurando la trascendencia, la superación, a nivel integralmente humano y no del físico tan sólo"; por tanto, asumimos que la Ciencia de la Motricidad Humana no estudia un ser que se mueve, ya que estudia un ser que se mueve con intencionalidad.

La Educación Física se preocupa por el estudio del desarrollo de las capacidades físicas del individuo. Los que centran sus estudios en investigaciones, única y exclusivamente, en las Ciencias del Deporte no salen de una parcela de la Motricidad Humana, desde ésta todo se refiere al cuerpo: al cuerpo-memoria y al cuerpo-profecía, al cuerpoestructura, y al cuerpo-conducta, al cuerpo-razón y al cuerpo-emoción, al cuerpo-natural y al cuerpo-cultura, al cuerpo-lúdico y al cuerpo-productivo, al cuerpo-normal y al cuerpo con necesidades especiales (Tavares, 2001).

No podemos definir la Motricidad sin recordar que ésta no solo se circunscribe a escenarios específicos e institucionalizados como la educación formal, llámese ésta escuela, colegio o universidad; sino que es parte de la vida misma del sujeto; es una capacidad más con la que todos los seres humanos nacemos y que se desarrolla al igual que el resto de capacidades en el diálogo con otros. Es decir, es la propia manifestación de la vida en sus distintos modos de expresarse e impresionarse en ese diálogo yo-otro-cosmos. Su intención es la toma de consciencia desde la vivencia (sensibilidad) y su fin o propósito es el desarrollo humano de cara a la transcendencia (Jaramillo, 2007).

A través de estas manifestaciones el hombre ocupa su lugar en la Naturaleza, no está contra ella, no es ni sobrenatural ni antinatural, sino que se mantiene en su lugar de persona. En estos términos, podemos suscribir que la Motricidad es una forma concreta de relación del ser humano consigo mismo, con el mundo y con sus semejantes, una forma de relación que se da a través de su corporeidad, de la acción humana, caracterizada por la intencionalidad y por el significado.

Entendemos "acción" desde los postulados de Blondel, el filósofo de la acción que destaca la palabra 'acción', sin excluir la idea de una inmediata y perfecta realidad, implica la distinción de tres fases y de tres significaciones discernibles, separables o no:

1. La acción indica primitivamente el ímpetu iniciador en lo que tiene de vivo y de fecundo, de productivo y de finalista a la vez, 2. La acción puede designar (allí dónde una operación discursiva y compleja se hace indispensable para que se realice) la serie continua y progresiva de los medios empleados: proceso necesario para la ejecución del designio inicial que debe recorrer el intervalo que separa el proyecto del efecto y, según la expresión escolástica, el terminus e quo del terminus ad quem, per gradus debitos. 3. La acción puede significar, finalmente, el resultado obtenido, la obra conseguida, la terminación realizada. Puede entonces considerarse este resultado menos como un objeto bruto que como una especie de creación viviente donde la eficacia y la finalidad han conseguido unirse valorando todas las potencias mediadoras que han servido para esa maravillosa innovación, evocada por esa pequeña palabra llena de misteriosas riquezas: obrar (cit. en Ferrater, 1958: 40). 
Para la Ciencia de la Motricidad Humana acción es: sensación-percepción-conscienciaemoción-pensamiento-energía-ejecución (RIIMH, 2006).

El término Motricidad surge de un corte epistemológico en la Educación Física. En el siglo XVII fue a través de una propuesta de John Locke, de la que surgió la denominada Educación Física, con el propósito de dominar y adiestrar el cuerpo. Locke expresaba el valor de la Educación Física en su trabajo titulado Some Thoughts Concerning Education. Para Locke, la Educación Física era un medio de preparar a las personas ante posibles emergencias de salud, que involucraban dificultades y fatiga. Además, este filósofoeducador vislumbraba a la Educación Física como forma de lograr el desarrollo de un cuerpo vigoroso y listo para cualquier acción que lo necesite.

Hemos de entender que para dar cuenta del pensamiento de John Locke con respecto a la educación, es necesario situarlo en su contexto y recordar que pertenece al siglo XVII, al tiempo de Descartes, Bacon, Berkeley y Hobbes; un momento en el que surge el racionalismo, el que hace necesaria la evidencia demostrativa. La actitud fundamental de Locke es empirista, siendo considerado como uno de los más distinguidos representantes de la corriente empirista inglesa (Gallo, 2006).

Aunque actualmente no es la concepción que se encuentra en el imaginario de sus profesionales, que asumen el valor humanizante de la Educación Física a pesar de no reconocer en su gran mayoría la incoherencia del término, en la escuela se ha de enseñar primero la condición humana, procurando que sus prácticas sean humanizantes (Trigo y Montoya, 2007). Quizás muchos profesionales se encuentren más próximos a la Motricidad que a la denominada Educación Física.

La Motricidad implica una nueva mirada de la Educación Física y del deporte, lo que conlleva un giro hacia una praxis que pone énfasis en lo humano de todas las actividades que realizan las personas, ya sean de trabajo o de ocio; lo que nos lleva a hablar no de movimiento humano y sí de acción humana.

Si hablamos de Motricidad explícitamente, Paulo Freire (1989) afirma que

cuando la práctica es tomada como curiosidad, entonces esa práctica despierta horizontes de posibilidad. Las personas entonces hacen de sus discursos un panorama de lo que es posible hacer. No se limitan apenas a aquello que debe de ser hecho. Las personas descubren con la práctica sus posibilidades. Mismo dentro de los límites analizados las personas organizan esfuerzos para viabilizar lo que está siendo difícil de hacer (Freire 1989: 34).

Esta visión de la práctica que muestra Freire se aproxima mucho a la praxis que defendemos desde la Motricidad, generando la necesidad de pensar la práctica, de fomentar la creatividad, la búsqueda de la superación y el pensamiento crítico.

En la evolución de la Motricidad, uno de los puntos importantes a desarrollar reside en la búsqueda de la máxima coherencia posible entre teoría y práctica (praxis). Uno de los pasos a subrayar es el que invoca la necesidad de hacer ciencia, que es una forma de desalienar (Manuel Sérgio) y de alfabetizar (Paulo Freire), y por tanto de conscientizar. De lo que inferimos la existencia de dos discursos que convergen en el interés común de desarrollar al ser humano y el poder sobre sí mismo; esto es sobre su vida y sobre su oportunidad de construir el mundo.

Hablar de Motricidad Humana es dirigirse al movimiento histórico humano, desde las prácticas corpóreas de lo cotidiano y las prácticas socialmente sistematizadas y acumuladas, como lo son las modalidades deportivas y de ocio. Supone admitir las dimensiones humanas: social, política, afectiva, cognitiva, instintiva, fluyendo en acciones 
singulares que revelan la naturaleza de la humanidad, su intervención en el medio y las influencias de éste sobre lo humano (Soares y Pernambuco, 2005). Por ello afirmamos que la Motricidad nos permite comprender y distinguir una vivencia de la otra; es decir, nuestra intencionalidad y sus consecuencias.

Para comprender el movimiento humano consciente, en sus diferentes manifestaciones, es necesario considerarlo siempre en el contexto de las relaciones sociales en las que sucede. Así, "el desarrollo de la consciencia y de la Motricidad específicamente humanas sucede en el ámbito de las formaciones sociales definidas y, por tanto, implica la transmisión cultural de la experiencia histórica del hombre" (Kolyniak, 1997:37). Esta idea se refuerza si, además, entendemos que el cuerpo no es solo forma anatómica y física, sino también sentido y significación, proceso de comunicación básico, no verbal y vital, centro de diálogo con el mundo social y contextual.

De ahí que sea necesario proponer unos principios en torno a los que debería girar la praxis de la Motricidad en sus diferentes manifestaciones o ámbitos de actuación, que se resumen en:

- Buscar siempre la acción intencional de la persona que se manifiesta.

- Partir de que la Motricidad forma parte de la vida misma de las personas e integrar esa práctica como tal.

- Centrarse en el valor humano de la acción y tener un sentido.

- No perder de vista el respeto por la condición y la dignidad humana, respetando los momentos evolutivos de las personas.

- Enriquecer todas las dimensiones humanas, social, política, afectiva, cognitiva, instintiva, permitiendo el fluir de la vivencia personal.

- Primar el aspecto lúdico en todas sus manifestaciones, incluyendo este valor en las diferentes manifestaciones competitivas que se puedan producir.

- Procurar la transcendencia del ser humano debe ser el sentido de su acción intencional.

- Finalmente, que toda acción motrícia debe tener presente el mantenimiento, respeto o mejora de la salud como valor.

Si entendemos que el deporte ha sido uno de los fenómenos más significativos del siglo XX, también hemos de entender que, en este tiempo, ha sido y es uno de los principales exponentes en los que se ha manifestado la Motricidad, ya que es el cuerpo en acción el que se manifiesta y expresa.

En la actualidad, hablar de deporte puede tener distintas consideraciones en función del contexto en el que se cite. Esto es novedoso, pues el deporte no ha tenido el mismo significado en distintas épocas históricas, ni en las mismas culturas (García, 2006). Según la Real Academia de la Lengua Española (1992), la palabra deporte tiene tres acepciones:

Recreación, pasatiempo, placer, diversión o ejercicio físico, por lo común al aire libre.

Ejercicio físico, por lo común al aire libre, practicado individualmente o por equipos, con el fin de superar una marca establecida, o de vencer a un adversario en competición pública, siempre con sujeción a las reglas.

Actividades físicas ejercidas como juego o competición sujeta a normas cuya práctica supone entrenamiento o buen estado físico (314).

Con estas definiciones, podemos reforzar nuestra primera alusión a este término, en lo que atañe a su conceptualización, siendo factible hablar de otros tres tipos de deporte: 
el de ocio y tiempo libre, el federativo y el profesional (Vázquez, 1998). El artículo 2 de la Carta Europea del Deporte del Consejo de Europa, también define el deporte como "todo tipo de actividades físicas que, mediante una participación organizada o de otro tipo, tengan por finalidad la expresión o la mejora de la condición física o psíquica, el desarrollo de las relaciones sociales o el logro de resultados en competiciones de todos los niveles" (cit. en Monrás, 2008: 4).

Lo que de alguna forma queda claro es que en estas concepciones, el deporte no es algo cerrado ni concreto ni se ciñe a prácticas tradicionales o más extendidas conocidas como tal (fútbol, atletismo, tenis, etc.); digamos que se habla en una misma definición de distintas actividades o de diferentes manifestaciones de un mismo fenómeno. Y entre otras cosas, hemos de destacar que en una sociedad donde parecen primarse los aspectos individuales, el deporte puede y debe configurarse como un elemento socializador. Así lo entienden autores como Heinemann (1999), que ponen énfasis en que el deporte actual presenta una serie de peculiaridades:

a) No hay límites naturales a la satisfacción.

b) Las aspiraciones son independientes entre sí.

c) Se puede observar una desinstitucionalización y una desregulación.

d) El consumo del deporte se complementa de otros elementos propios del tiempo libre (58).

Cuando hablamos de deporte, ponemos de manifiesto nuestro propio concepto, en el que se busca mejorar la calidad de vida de las personas en todas sus dimensiones (psico-socio-afectivo-motriz) y en todas las manifestaciones del deporte, como son sus vertientes educativa, de salud, de ocio o de competición, partiendo siempre de unos principios éticos y de respeto de unos valores que no sean lesivos para los individuos.

La literatura existente aporta aproximadamente veinte contenidos figurativos distintos del término deporte, la mayoría de ellos, fruto de un proceso de investigación que ha requerido previamente el establecimiento de unos límites que guíen la atención del investigador hacia los fenómenos que deben ser observados (García de la Torre, 2006).

El concepto de deporte debe responder a una lógica de enculturación (Solar, 2007) y el deporte local no debe centrarse únicamente en modas que vayan surgiendo, pues dentro del patrimonio cultural de todos los pueblos existe una Motricidad que se manifiesta en danzas, modalidades deportivas, juegos, exhibiciones, etc.

De ahí que cuando aludimos al deporte local, hacemos referencia a la necesidad de promocionar el deporte en todas sus dimensiones educativas y culturales, tratando de presentarlo como una actividad deseable, positiva y que contribuye al buen vivir. Por ello, en una sociedad cada vez más "virtual" debemos buscar el punto de empatía, que consiga atraer a las personas que se derivan convenciéndolas de los beneficios de la interacción deportiva, al implicar juego, relación, interacción, mostrarse uno mismo, etc. Y que al tiempo, también ayuda a superar el sedentarismo, cada vez más presente en nuestras sociedades.

Aunque cuando hacemos referencia al deporte desde la Ciencia de la Motricidad Humana, nos referimos a un deporte en el que la competición es también diálogo y convivencia: un tiempo en el que los participantes se necesitan para vivenciar un momento de placer, en el que sentirse libre en el respeto de las normas, apreciando la transcendencia del tiempo lúdico, como un deporte que se integra en el derecho al ocio de las personas y una manifestación de la salud, para generar y mantener cultura, que se arraiga en lo más profundo de la vida de las personas y comunidades. 
Un deporte pensado por los practicantes, es algo más que técnica y se convierte en un factor de educación de la comunidad, sin olvidar ninguna de sus manifestaciones (desde la alta competición hasta el deporte para todos), carente de violencia y sin barreras, un espacio de convivencia en el que no hay razas, ni países, ni clases sociales; como afirma Sérgio (1991: 31): "un deporte-alternativa, en lugar de un deporte-repetición".

Contemplar el Deporte desde la Motricidad, supone tomar en consideración el deporte que se practica en la sociedad actual; como nos dicen Trigo y Montoya (2009: 13): "solo hay progreso deportivo cuando la cualidad precede a la cantidad, cuando al tener se antepone el ser. Lo que vale decir que el problema crucial en que se debate el deporte no está en la opción entre continuar o impedir el progreso que está ahí, sino en orientarlo en otra dirección". La diferencia se encuentra en el punto de vista con el que se observa el fenómeno, pues desde la Motricidad se aboga por una práctica en la que la finalidad es el desarrollo del ser humano y no el ganar-perder de esa práctica deportiva, de modo que es un deporte en el que sin olvidar el rendimiento, es respetuoso con la condición humana. Este aspecto de la salud está adquiriendo mayor relevancia debido a las continuas variaciones dietéticas que se están implantando y que suelen dejar la salud en un segundo plano.

Uno de los principales elementos a tener en cuenta sobre el desarrollo personal a través del deporte, es la creatividad, pues es en ella donde generamos un espacio para aportar momentos de innovación, de juego, libertad, momentos de brillantez y de excelencia. Y en todo ello está presente la Motricidad y la esencia de las personas.

\section{LA MOTRICIDAD HUMANA EN CLAVE EDUCATIVA}

La dimensión pedagógica de la Motricidad conlleva referirse a los contenidos educativos y como tal implica tener en cuenta sus aspectos didácticos; en este sentido, tomamos como referencia la propuesta de educación libertadora de Paulo Freire, de modo que la base didáctica de la Motricidad debe estar asentada en el desarrollo de la creatividad (Kon-traste y Trigo, 2000).

Con este enfoque, asumimos el compromiso de coherencia que han de tratar de satisfacer los docentes de la Motricidad, pues para basar nuestra pedagogía en la creatividad, necesariamente hemos de plantearla como una tarea cotidiana, ya que enseñar supone a menudo contagiar al otro a través del ejemplo, por la presencia y por la acción. Los alumnos/as, deportistas, colegas, etc., conectarán o alcanzarán mayor grado de empatía si lo que se muestra en teoría es parte de aquello que somos en las demás dimensiones de nuestro ser. Como dice Freire (1989: 33): "el cuerpo nos remite a los conocimientos que se organizan en el interior de las acciones, de las prácticas de vida”.

Nuestra perspectiva cuando hablamos de Motricidad, no supone destacar una dimensión pedagógica que se distinga por sí misma de la Motricidad, pues aunque existen diferentes teorías que intentan hacer avances en este aspecto -por ejemplo, evolucionando desde la Educación Física a la Educación Motora (Manuel Sérgio) o a la Paidomotricidad (Equipo Kon-traste, 2005), u otras posibles denominaciones-, estos intentos caen en el error del dualismo que tanto critican; así, entendemos que si el elemento a estudiar es la Motricidad y esta explica las diferentes manifestaciones motrícias de la corporeidad, será a través del desarrollo de sus propios elementos didácticos como los profesionales 
de la Motricidad podrán hacer sus contribuciones para conseguir un desarrollo personal más humano e íntegro, en el que se respeten los diferentes ritmos de evolución de las personas en las distintas fases de su vida.

Llegados a este punto afirmamos que la Motricidad es el campo de estudio que se encarga de comprender y contribuir a mejorar el desarrollo de la corporeidad en los diferentes contextos y sociedades en las que vive el ser humano; y que, como tal, abre un campo profesional, tradicional y parcialmente ocupado por los profesionales de la conocida Educación Física, de los profesionales del Deporte, de la Fisioterapia o de la Kinesiología.

No creemos, desde lo que venimos manifestando, que la Motricidad como capacidad humana y como ciencia que la estudia, pertenezca a una determinada área de conocimiento. Pensamos que el campo profesional y de estudio de la Motricidad Humana le corresponde a cualquier ámbito científico y profesional que estudie al ser humano en su acción consciente de buscar la transcendencia.

En la actualidad, muchos profesionales actúan en su día a día con esta perspectiva aunque no lo hagan explícito, si bien hay otros muchos profesionales de campos afines a la Motricidad que trabajando con personas en acción, no buscan el desarrollo humano, ni la transcendencia, centrándose más en resultados deportivos y otros logros.

Sérgio (2008) reclama una Educación Deportiva en la procura de una sociedad y un deporte diferente, asentados en la Ciencia de la Motricidad Humana, planteando una educación problematizadora (Paulo Freire). Esto no hace sino reforzar esa necesidad de cambio que venimos anunciando en cuanto a la práctica de la actual Educación Física, necesitada de una fundamentación teórica que la dote de sentido y que consiga penetrar en el imaginario social de la comunidad.

En lugar de hablar de una dimensión pedagógica y dar un nuevo nombre, entendemos que la Motricidad como objeto de estudio ha de tener su propia Didáctica, y como tal su propia Pedagogía, que además se distingue de la Didáctica de los demás campos de estudio por tener sus propias características. Entre otras, se distingue porque:

- Es una praxis integradora, que pone de manifiesto en cada momento todas las dimensiones del ser humano (psicológica, sociológica, afectiva, motricia).

- Busca la transcendencia a través de sus diferentes propuestas y manifestaciones.

- Usa como base de acción el desarrollo de la creatividad de las personas.

- Es capaz de unir la superación personal con la dimensión lúdica del ser humano en todas las propuestas.

- Muestra una praxis ecológica de respeto a las personas y al medio ambiente.

- En su desarrollo, no se distinguen diferencias por condición social, raza, intelecto, ni de ningún otro tipo.

- Ha de ser una praxis optimista y alegre, que llene de vitalidad y energía a las personas que la vivencien.

En estos aspectos identificativos, se muestra lo que entendemos como los principios que debe buscar la dimensión pedagógica en toda propuesta de desarrollo de la Motricidad, ya sea en la escuela, en la calle, en la playa, etc. Y con cualquier grupo de personas, independientemente de su edad o condición.

Antes de avanzar en la idea del profesional de la Motricidad, hemos de insistir en la necesidad de que ésta como valor tenga una adecuada correspondencia en los conceptos, pues el cambio no debe ser únicamente de nombre, sino que debe ir acompañado de nuevos significados y de una adecuada praxis diferenciadora. 
Este cambio lo describe Sérgio (cit. en Trigo y Montoya, 2007), cuando señala que: la ciencia de la Motricidad humana exige el paso, en la investigación y en las aulas y en los entrenamientos y en las competiciones y en las técnicas de salud, del cuerpo-objeto al cuerpo-sujeto (yo soy mi cuerpo) y al fin de todos los dualismos tradicionales: cuerpo-espíritu, hombre-mujer, señor-siervo, etc. El cuerpo no se reduce a simple organismo, él es una red de intencionalidades, un horizonte de posibilidades, la fuente de comunicación con el otro (31).

Es necesario que seamos capaces de desarrollar nuestra propia praxis, de activar o habilitar vivencias que provoquen situaciones que obliguen a las personas con las que trabajamos a pensar, a desplegar su creatividad, a ser más conscientes de sí mismos, y que provoquen su propia evolución personal y social que será la antesala de un cambio en el imaginario social de nuestra comunidad, y un giro en cuanto a los valores sociales existentes, desarrollando así no solo la consciencia de uno mismo, y a la vez unos valores de respeto, convivencia y cultura comunitarios.

Creemos necesario destacar que los profesionales de la Motricidad, en su desempeño diario, cuando trabajan con personas, además de pensar, hacer, sentir o querer, deben analizar el contexto social, económico, educativo y ambiental que engloben la verdadera realidad del ser humano, dándole sentido a ese movimiento intencional de superación y/o transcendencia. La Motricidad se inscribe en la vida cotidiana, al igual que la educación, pues forma parte de la evolución y el desarrollo humano. Por lo tanto, no deberíamos hablar de corporeidad sino de corporeidades, no de Motricidad sino de motricidades, no de lenguaje, sino de lenguajes, etc.

La capacidad de autopercibirnos y ser conscientes de nuestra propia corporeidad, deberá posibilitar experiencias que incidan directamente en lo más profundo de nuestro emocionar, y ello nos ayudará sin duda a autoanalizarnos de forma que podremos conocernos mejor, conocer mejor a los demás y así tener mayor facilidad para empatizar con nuestro entorno.

Sin duda, esta coherencia personal podrá ayudarnos a vivir sin temor a expresarnos, a afrontar nuestros retos personales, y a activar cierto inconformismo con lo que somos o tenemos, que también es propio de los seres humanos. Todas estas cuestiones se encuentran en mayor o menor medida limitadas (condicionadas) por nuestro entorno más inmediato, pues es el que nos ofrece las posibilidades, aunque referirse a entornos cercanos, aludir a distintas manifestaciones del espacio físico y hasta virtuales, como ámbitos o contextos con los que mantenemos algún tipo de relación, en la que habitamos y convivimos.

Los retos personales, los miedos, las costumbres y los hábitos, los situamos en este contexto. Una de las manifestaciones que consideramos propia de una verdadera praxis que ayude a evolucionar en lo personal es el optimismo, la visión positiva de quienes somos y del mundo, la creencia en la posibilidad de crear un mundo mejor, una salud global y globalizada, que favorece la posibilidad del cambio y del crecimiento.

Vivimos una realidad presente, pero una verdadera visión desde la Ciencia de la Motricidad Humana implica mostrar un horizonte de utopías realizables, ya que nadie más que nosotros es capaz de hacer un sueño realidad, pues no existe ningún gran invento, ni ningún gran logro de la humanidad, que no haya sido soñado por alguien antes.

Por tanto, afirmamos que una verdadera praxis de la Motricidad debe empezar por una percepción optimista, llena de retos e integradora del yo con el medio, buscando formar parte de esa realidad global y compleja. 
Así, en el plano personal, estos son los principios de coherencia que tratamos de aplicar en nuestro día a día, en medio de la compleja realidad que nos rodea, centrada principalmente en el valor de tener y tener más cosas. Una realidad en la que muy pocos son conscientes del daño que la producción sin límites produce al medio natural y a las personas, pues "el nuevo modelo de sociedad debe rehacer el tejido social a partir de las múltiples potencialidades del ser humano y de la propia sociedad" (Boff, 2000: 38).

Esta coherencia personal, y el respeto a las personas y al desarrollo humano, el trabajo desde la creatividad propia y aplicada en nuestro día a día, es lo que debe distinguir al profesional de la Motricidad en relación a otros. Y es ése precisamente el tipo de profesional que estamos buscando, implicado con su vida, con su contexto y con su quehacer profesional, capaz de buscar la transcendencia en el día a día, trabajando en todo momento a través del movimiento consciente.

En este sentido, hemos de destacar que la Motricidad construye una visión del hombre simultáneamente carente y trascendente, hacedor de sí mismo y de su humanidad en un proceso consciente de reencantamiento. El profesional de Motricidad Humana es consciente del desafío que supone re-encantarse y re-encantar el mundo. En donde haya personas hay desencantos y desencuentros producidos por un paradigma rígido y determinista (Feitosa et al., 2006). Este debe ser uno de los principios de todos los profesionales de la Motricidad independientemente del campo en el que desarrollen su labor (entrenamiento, educación, salud, organizaciones, vida cotidiana, etc.), que es la creación de nuevos espacios de convivencia; y se deben encontrar en las intencionalidades de su trabajo, sin olvidar su campo de acción.

El perfil del profesional de la Motricidad, debe ser el de alguien capaz de comprender que todas las manifestaciones motrícias están dotadas de significado e implican una dimensión ética, capaz de hacer uso de unos principios básicos y unos métodos característicos que faciliten el autoaprendizaje y el desarrollo personal, potenciando la capacidad creativa y el esfuerzo en las personas. Capaz de comprender el verdadero potencial de la Motricidad en el desarrollo humano, de tener una capacidad de autocrítica que le permita igualmente crecer y transcenderse a sí mismo en el encuentro de su coherencia interna. Podemos tomar como resumen las siguientes palabras: "un especialista en 'Motricidad Humana' es un especialista en 'desarrollo humano' y por lo tanto en empatía, desde sus distintos niveles y distinciones" (Toro, 2005: 135).

Hacer referencia a la gestión de cualquier tipo de actividades desde la Administración Pública, y concretamente a la municipal, por ser ésta la Administración más cercana al ciudadano, supone comenzar por preguntarnos cuál es la tarea del municipio, qué funciones debe cumplir con los miembros de la comunidad. En este contexto, y entre otras cuestiones, podemos plantear: ¿cuál es el papel del Estado? ¿generar patrones? ¿educar? ¿enseñar a convivir?

Las respuestas a las preguntas anteriores, mostrarán nuestras ideas sobre las funciones del Estado y las demás Administraciones Públicas con respecto a su papel en el desarrollo humano. Aun así, cuando hablamos de gestión desde la perspectiva de la Motricidad hemos de comenzar por la familia, ya que la Motricidad es contextual y la familia es el primer agente promotor de las acciones humanas, ya sean deportivas, culturales, etc. Ocupando en este caso la escuela un papel secundario, pues para los niños/as el primer modelo a seguir suelen ser sus padres u otros familiares cercanos los que los acompañan en sus primeras fases de crecimiento y desarrollo. 
Al explicar la gestión deportiva desde la Motricidad, necesariamente hemos de tener en cuenta el contexto, y eso implica contar con los cuatro elementos principales que se encuentran en la comunidad: la familia, la escuela, las asociaciones y la Administración (municipio). Estos deben de convertirse en promotores y gestores activos desde los inicios, pues realmente han surgido de la necesidad social de gestionar las diferentes estructuras sociales, ya sea en la vida personal, en la transmisión cultural y el desarrollo educativo, en la gestión del tiempo libre, o en el establecimiento de unas normas mínimas de convivencia y respeto. En todos estos aspectos se puede influir desde cualquiera de las estructuras sociales.

La escuela (y el deporte) puede ser un factor de cambio y transformación social, ayudando a formar especialistas y técnicos, que sean ciudadanos libres y liberadores (Sérgio, 2008). Por eso afirmamos que el sistema educativo y el sistema deportivo son subsistemas del sistema social, y así la escuela (y el deporte) está condicionada por lo que ocurre en la sociedad entera.

Los municipios son o han de ser el lugar de encuentro de los ciudadanos: en ellos se puede y se debe incentivar la participación ciudadana, y esa no es una función solo política, ya que, aunque los responsables políticos marcan las pautas a seguir, son los técnicos municipales los que deben diseñar y poner en práctica verdaderos programas de participación, fórmulas para llegar a las personas y promover la generación de cultura, hasta el punto de que no debería existir ninguna acción cultural local en la que no estuviesen implicados todos sus miembros.

Las personas han de sentirse responsables de su propia formación, y son los técnicos municipales los que intervienen (o así debe ser) como guías que estimulan la realización de actividades dotadas de sentido en el seno de la comunidad, incentivando la participación y la creatividad de los vecinos. La identidad es uno de los objetivos implícitos, tanto en la creación y mantenimiento de costumbres culturales, como en la participación en las actividades que se van generando. Así pues, desde el contexto de la Administración Local hemos de tratar de llegar al conjunto de la ciudadanía, atendiendo a los intereses que se consideren más oportunos.

El área principal en la que los Ayuntamientos pueden realizar una gestión desde la perspectiva de la Motricidad, es el área de deportes, que si bien por ley tiene entre sus objetivos potenciar el deporte en la edad escolar, no es menos cierto que entre los roles de la promoción del deporte está incluida la totalidad de la población; y es en el Deporte para todos donde debe centrar los esfuerzos: en un deporte que sea integrador para todos, participativo, cooperativo, generador de hábitos, de compañerismo y de inclusión social. Generaremos así una identidad saludable, donde personas de todas las razas y niveles sociales tendrán su espacio común, un espacio de convivencia entre iguales, en el cual las diferencias las marcaría el grado de implicación o de habilidad, o el sentido lúdico, en ese impregnarse de la actividad que nos facilite la transcendencia.

El municipio puede y debe potenciar estas acciones favoreciendo el desarrollo de actividades propuestas por los propios vecinos, facilitando los medios y recursos que se puedan disponer desde el municipio, con colaboraciones puntuales, a través de subvenciones, etc.

Una gestión municipal desde la perspectiva de la Motricidad, debe incluir la preocupación por el desarrollo humano, el mantenimiento y la generación de la cultura, convirtiendo así la gestión en algo contextual, como se destaca del propio paradigma de 
la Motricidad. Ello debe implicar el trabajo en equipo, procurar la participación activa más que el espectáculo, considerando grandes logros, los que, además de implicar la participación, conlleven la satisfacción y posible generación de hábitos; lo que a su vez implica el desarrollo de la capacidad crítica y de pensamiento de los miembros de la comunidad.

Las personas deben tener un papel central y principal en la gestión, pues los recursos municipales deben habilitarse por y para la utilización de los vecinos, sin que ello implique una relación de dependencia; más bien todo lo contrario, esta gestión debe orientar a los vecinos a ser capaces de autogestionarse y a promover su propia autonomía.

No debe obviarse que el deporte es una práctica que exige una formación, una pedagogía, un espacio en el que se permita que afloren todas las características del ser humano y a partir de ahí se puedan enfocar en la búsqueda de la superación. Pues como fenómeno social, el deporte es humanizante, y así lo debemos entender y mostrar los profesionales y/o responsables de su gestión.

En este sentido, hay que destacar que una gestión comunitaria, desde la perspectiva de la Motricidad, ha de implicar la promoción y el logro de un mayor desarrollo humano, en el que los servicios a los ciudadanos sean también servicios de los ciudadanos, siendo estos cada vez más autónomos y capaces.

Para conseguirlo los Ayuntamientos han de desarrollar planes y programas que procuren la autonomía y la capacidad de autogestión, favoreciendo que las Administraciones Locales puedan asumir cada vez más una función de control, apoyo y promoción de la salud, la calidad de vida y la buena convivencia. Ello supone buscar espacios y momentos, que promuevan una consciencia comunitaria a través de la que las personas procuren la mejora social y el desarrollo de las comunidades que habitan.

Hablamos de un profesional de la Motricidad necesariamente comprometido con su trabajo, con su forma de vida, y que en toda su intervención busque que las personas a través de la acción consciente procuren la superación hacia la transcendencia. Pero realmente lo que tratamos desde la Motricidad es la autoconstrucción de lo personal y de lo social, de ahí que entendamos que "el hombre es un ser carente, consciente de su carencia y por eso un ser práxico, eminentemente cultural y, por tanto, transformador del mundo, de modo a resolver sus carencias" (Feitosa et al., 2006: 61).

El proceso de construcción de conocimiento de la $\mathrm{CMH}$, implica variadas formas de investigación e intervención que, en su conjunto, se configuran como praxis y, como tal, posibilita la emergencia de un "modus operandi" que puede caracterizar un profesional con identidad propia. Por tanto, la Ciencia de la Motricidad Humana puede componer la formación académica y profesional en las más diversas áreas de intervención, en niveles de pregrado y posgrado.

La Ciencia de la Motricidad Humana puede ser objeto de la formación de un profesional cuya especificidad consiste en el dominio del conjunto sistematizado del conocimiento producido en este área. Este profesional no debe sustituir cualquier otro ya formado en otras áreas de intervención (Educación Física, Fisioterapia, Terapia Ocupacional, Psicología, etc.). Su campo de actuación será construido en la medida en que sus conocimientos y sus acciones fueran reconocidas como relevantes para la satisfacción de determinadas demandas sociales, a partir del diálogo interdisciplinar.

En este sentido, podemos hablar del debate en torno al concepto de Motricidad, ya que dependiendo del contexto en el que se use tiene una significación diferente; esto 
no se debe al origen del término, ni incluso a los profesionales que hablan de ella, ya sea para criticarla o para tratar de mostrar sus potencialidades. La cuestión es que en el imaginario social, la palabra Motricidad es sinónimo de movimiento. En muchos círculos profesionales afines a la Educación Física, sucede lo mismo, y éste es un cambio que puede durar algunas generaciones, de llegar a producirse.

En otras áreas de conocimiento, como la Psicología, adoptaron el término y lo redimensionaron varias veces, hablando de Psicomotricidad, Psicomotricidad Relacional, Sociomotricidad, etc., pero sin ir más allá en la acepción del término. En relación a este hecho, Manuel Sérgio desarrolla el concepto orientado a un ser humano complejo, en un movimiento operante de búsqueda de la transcendencia, presentándolo como el fin del dualismo cartesiano y como la emergencia de una nueva Ciencia, la de la Motricidad Humana, que podrá constituir por sí sola un área de conocimiento.

¿Es un problema de falta de discusión? Probablemente nos estamos enfrentando a una propuesta de cambio de cierto relieve, que implica superarnos y no resulta fácil comenzar a hablar de ciencia encarnada (Trigo, 2011), ya que supone el atrevimiento de introducir algo nuevo en un concepto de ciencia muy arraigado; en estudiar algo que en la mayoría de los casos no es observable y que implica hablar de integración metodológica y no de mediciones de tiempos, de distancias, de parámetros fisiológicos, etc., perfectamente medibles con las nuevas tecnologías. Investigar en Motricidad Humana supone una búsqueda del bienestar y un intento de construcción de una persona más autónoma, autocrítica y con un gran potencial de superación y transcendencia.

Desde esta perspectiva de la Motricidad, hemos de entender el deporte como acción intencional, como cultura moderna, como teoría y como invención; en estas circunstancias, la práctica de la Motricidad es una condición para la construcción de la consciencia, de modo que esta altera la propia Motricidad (Kolyniak, 1997).

\section{REFERENCIAS BIBLIOGRÁFICAS}

Benjumea, M. (2010). La motricidad como dimensión humana - Un abordaje transdisciplinar. España-Colombia: iisaber.

Berman, M. (2004). El reencantamiento del mundo ( $9^{\mathrm{a}}$ Ed. ed.). Santiago de Chile: Cuatrovientos editorial.

Boff, L. (2000). La dignidad de la tierra: Ecología, mundialización, espiritualidad. La emergencia de un nuevo paradigma. ( $1^{\mathrm{a}}$ ed.). Madrid: Trotta.

Bohórquez, F., y Trigo, E. (2006). Corporeidad, energía y transcendencia. Somos siete cuerpos (Identidades o notas). Pensamiento educativo, n.38, 75-93.

Brandão, K. (2001). Para a unificação em Ciência da motricidade humana (1 ed. Vol. 1). Natal: UFRN.

Capra, F (1982). O ponto de mutaçao. A ciéncia, a sociedade e a cultura emergente. Sao Paulo: Cultrix.

Cyrulnik, B. y Morin, E. (2005). Diálogos Sobre la Naturaleza Humana. Barcelona: Paidós.

Feitosa, A., Kolyniak, C., y Rath, H. (2006). Mudanzas:Horizontes desde la Motricidad. Popayán: Universidad del Cauca.

Freire, P. (1989). Que Fazer: Teoría e prática em educação popular (8 ${ }^{\mathrm{a}}$ ed. ed.). Petropolis: Vozes.

Gallo, L. (2006). El pensamiento educativo de John Locke y la atención a la Educación Física. Educación Física y Deporte, vol. 25. n.1, 97-113. 
García de la Torre, S. (2006). El deporte para todos desde una perspectiva provincial o territorial. En Kait (Ed.), Deporte, gestión y municipio. Aspectos clave. (Vol. 1, pp. 313-327). Bilbao: Eudel. Heinemann, K. (1999). Sociología de las organizaciones voluntarias. El ejemplo del club deportivo. Valencia: Tirant lo blanch.

Jaramillo, L. G. (2007). Motricidad Humana ¿Cultura del cuerpo o cuerpo cultural? Motricidad y Persona, vol. 1, n. 3, 45-53.

Kolyniak, C. (1997). O esporte como objetivo da Educação Física ou da ciencia da Motricidade Humana. Discorpo, n.7, 31-46.

Kolyniak, C. (2005). Propuesta de un glosario inicial para la ciencia de la motricidad humana. En U. d. Cauca (Ed.), Consentido (pp. 29-39). Popayán: Universidad del Cauca.

Kon-moción, G., Trigo, E., Naudorf, G., Yanza, P., Bohórquez, F. y Córdoba, C. (2007). Para los nuevos desafíos: Programa de motricidad humana y gestión comunitaria. Paper presented at the V Congreso internacional de Motricidad humana, Valdivia. Chile.

Kon-traste, y Trigo, E. (1999). Creatividad y Motricidad. Barcelona: Inde.

Kon-traste, y Trigo, E. (2000). Fundamentos de la Motricidad ( $1^{\mathrm{a}}$ ed.). Madrid: Gymnos.

Merleau Ponty, M. (1999). Fenomenolgia da percepcão (C. A. R. d. Moura, Trans. $2^{\mathrm{a}}$ ed.). Sao Paulo: Matins Fontes.

Monrás, J. (2008). Cooperación deportiva municipal: Expectativas de futuro. Paper presented at the Jornadas SID Tecnodeporte, Zaragoza.

Real Academia Española (1992). Diccionario de la lengua española. (21 ${ }^{\circ}$ ed.). Madrid: Espasa Calpe.

RIIMH. (2006). La Ciencia de la Motricidad Humana (CMH) cómo área autónoma de conocimiento: Trayectoria desde la Red Internacional de investigadores en Motricidad Humana. Consentido, 6.

Sérgio, M. (1991). A pergunta filosófica e o desporto. Lisboa: Compendium.

Sérgio, M. (1999). Um corte epistemológico: Da Educação Física á Motricidade Humana (2ºd.). Lisboa: Instituto Piaget.

Sérgio, M. (2007). Algunas miradas sobre el cuerpo. Popayán: Universidad del Cauca.

Sérgio, M. (2008). Textos Insólitos ( $1^{\mathrm{a}}$ ed. Vol. 1). Lisboa: Instituto Piaget.

Soares, M. G., y Pernambuco, M. M. (2005). Emergencia disciplinar: cuando los saberes dialogan. En U. d. Cauca (Ed.), Consentido (pp. 149-155). Popayán: Universidad del Cauca.

Solar, L. (2007). Deporte y Municipio. ¿Hacia donde vamos? Retrieved 11/04/2009, from http:// www.munideporte.com/administracion/documentacion/ficheros/20090116130336luis_solar.pdf

Tavares, G. (2001). A temperatura do corpo. Lisboa: Instituto Piaget.

Toro, S. (2005). Desarrollo Humano y Motricidad: una aproximación desde la empatía. En U. d. Cauca (Ed.), Consentido (pp. 127-139). Popayán: Universidad del Cauca.

Trigo, E (2005). Ciencia encarnada. En U. d. Cauca (Ed.), Consentido (pp. 39-55). Popayan: Universidad del Cauca.

Trigo, E., y Montoya, H. (2007). Aportes de la Motricidad Humana a la Educación Física. Motricidad y Persona, vol., n.1, 9-45.

Trigo, E., y Montoya, H. (2009). El deporte desde la mirada de la Motricidad Humana. Motricidad y Persona, n. 6, 9-23.

Trigo, E. (2011). Ciencia e investigación encarnada. España-Colombia: iisaber.

Vázquez, F. (1998). Asociacionismo y voluntariado:Elementos colaboradores de programas deportivos. En FEMP (Ed.), El Deporte en Diputaciones y pequeños municipios (1 ${ }^{\mathrm{a}} \mathrm{ed} ., \mathrm{pp}$. 71-109). Madrid: FEMP. 
